\title{
XXXIV.- On the direction and mode of propagation of the electric force traversing interposed media
}

\author{
George J. Knox Esq. A.M. M.R.I.A.
}

To cite this article: George J. Knox Esq. A.M. M.R.I.A. (1840) XXXIV.-On the direction and mode of propagation of the electric force traversing interposed media, Philosophical Magazine Series 3, 16:102, 185-191, DOI: 10.1080/14786444008650017

To link to this article: http://dx.doi.org/10.1080/14786444008650017

册 Published online: 01 Jun 2009.

Submit your article to this journal $\lceil\pi$

Џll Article views: 2

Q View related articles $₫$ 
On the Electric Force as traversing Interposed Media. 185 to be the roots of the equation in that case: then the waves of which the lengths are $\frac{2 \pi}{k_{1}}, \frac{2 \pi}{k_{2}}, \frac{2 \pi}{k_{3}}, \ldots$ will be transmitted without absorption, and consequently will form a number of bright lines in the spectrum, yet probably too few to afford, by themselves, any sensible light. Now suppose $\varepsilon$ to decrease gradually, then all the roots $k_{1}, k_{2}, k_{3} \ldots$ will vary, but not with equal rapidity. Some of them may be changed in magnitude considerably by a very small change in $\varepsilon$, and, consequently, in the parts of the spectrum to which these roots respectively correspond, there will be bright bands. Other roots may be only slightly affected by a considerable change in $\varepsilon$; hence there will be, in the parts of the spectrum which correspond to these roots, rapid variations in the intensity of the light, producing dark bands or dark lines.

Perhaps the equation (42.) which we are considering, may, in certain cases, be much simplified; but I cannot proceed with the subject any further in the present paper.

I am, Gentlemen, yours, \&c.,

Littlemoor, Clitheroe, Feb. 6, 1840.

JoHN ToveY.

P.S. In my last paper, vol. xv. p. 451, last line but three, for increasing indefinitely read increasing or diminishing indefinitely, - p. 452, line 14, for $\cos m i=\sqrt{-1} \cdot \sin m i$ read $\cos m i+\sqrt{-1}$. $\sin m i ;-p .45$, line 28 , for $\rho, \alpha$, read $p_{1} \alpha_{1}$; and line 29, for $\alpha$, read $\alpha_{1} ;-$ p. 454, line 21 , for (23.) read (33.); lines 22 and 23 , for $e^{e x}$ read $e^{\epsilon x}$

XXXIV.-On the Direction and Mode of Propagation of the Electric Force traversing Interposed Media. By Geonge J. Knox, Esq., A.M., M.R.I.A.*

WHATEVER theory be adopted to explain the passage of the electric force traversing an intervening fluid or solid substance not undergoing electrolyzation, - whether we sup. pose it to originate in an inductive influence affecting the circumambient æther of each particle of the substance in the line of direction of the force, in whose alternate states of induction and equilibrium consists the passage of the electric current, (the rapidity of such changes constituting its intensity,) while the vibratory motion produced in the particles of the ather on each successive return to a state of equilibrium causes the

* From the Transactions of the Royal Irish Academy, vol. xix. 
phænomena of the light and heat developed; or whether we adopt the gross conception of the passage of a fluid; still it is important to determine if the electric force passes along the surface of the interposed substance, or through the interior of its mass.

Dr. Faraday* has shown that water will convey a feeble current of electricity, without undergoing electrolyzation. To determine whether, under such circumstances, it will convey an electrical current along its surface or through its substance, a glass tube, ten feet long, and half an inch internal diameter, bent in the centre twice at right angles, was filled with distilled water. Two copper wires, twenty feet long, having platina wires soldered to their extremities, were inserted in barometer tubes of six feet in length, the platina wires being sealed in the tubes within half an inch of their extremities. The other ends of the copper wires were connected with a delicate galvanometer, and a constant battery of successively one, two, four, \&c., pair of elements.

On immersing the platina wires in the liquid, their relative distances from each other should decrease if the current passes through the water, but should increase if it passes along the surface, the deflexion of the galvanometer indicating the path. With one pair of elements there was no deflexion of the galvanometer; with two pair of elements there was a slight deflexion visible through a lens, which increased slightly on immersing the platina wires in the liquid. With four pair of elements, a deflexion of two degrees took place when the platina wires were on the surface of the water; a deflexion of four degrees when they were immersed to the bottom of the tubes. As the number of alternations in the battery increased, so did proportionably the comparative deflexions of the galvanometer; the experiments proving that water, whether undergoing electrolization or not, conveys an electric current through its substance, and not along its surface, and that the decomposition of the water is an effect produced by the passage of the electricity when of sufficient intensity, and not the necessary consequence of its passage.

A similar experiment having been tried with phosphorus melted under spirits of wine, (being a non-conductor,) it was found to obey the same law with water; that is, to convey the current through its substancef.

To determine whether the metals followed the same law, I suspended from the top of the new patent shot tower at Wa-

* Series VIII. (970.)

$\dagger$ It was unnecessary to try similar experiments with the analogous bodies, sulphur, selenium, and iodine. 
terloo-bridge a leaden pipé, 170 feet long, and three-fourths of an inch internal diameter, through which was drawn an insulated copper wire, 180 feet long, one extremity of which being soldered to the inside of the end of the pipe, this end was sealed with fused metal, and to its external surface was soldered a copper wire of the same length as the former; round the tube, at its orifice, was twisted a copper wire ten feet long. The insulated wire being connected with a constant battery of one pair of elements in contact with one pole of an exceedingly delicate galvanometer, (constructed by Mr. E. M. Clarke of the Lowther Arcade, the other pole of the galvanometer was brought successively in contact with the extremities of the uninsulated wires. The deflexion was greater when the current passed along the wire connected with the orifice of the tube, (although here the contact was not so good,) than when it passed along that soldered to the sealed extremity.

Again, the uninsulated wires being connected with separate galvanometers, so as to aliow the current of electricity to pass along either of the uninsulated wires alone, or to be distributed between both, it was found (as well as could be determined by transposing the galvanometers, ) to have divided itself into two equal currents flowing along both wires.

From the first experiment we may infer that a current of electricity passes with greater facility along the surface of a metal than through the interior of its mass, although we cannot hereby infer that it could not pass through the interior of the metal, when this is the only road open for its transit*.

To the experiments with phosphorus it might be objected that its capability for conducting an electric current is due to the presence of water, of which some have supposed that it could not be entirely deprived, although the experiments of Sir H. Davy, wherein he obtained hydrogen and oxygen from sulphur and phosphorus by heating them in contact with potassium and sodium, and by submitting them to the electrolytic action of a powerful galvanic battery, did not prove that they were united with the basis of these substances in such proportions as to form water, nor indeed does he appear to have entertained such an opinion himself. His opinion of the na-

* The high conducting power of mercury for electricity renders it almost impossible to determine, by this method, whether metals in the fuid state obey the same laws of conduction as when in the solid state. If they do not, it is highly probable there is a general law, that all solids conduct along their surface, and all fluids through their substance. The investigation of such general law I propose to continue in another paper. 


\section{Mr. G. J. Knox on the Direction and Propagation}

ture of sulphur was, that it was "a compound of small quantities of oxygen and hydrogen, with a large quantity of a basis, that produces the acids of sulphur in combustion, and which, on account of its strong attraction for other bodies, will probably be difficult to obtain in its pure form*." To put the question beyond any further doubt, I will mention some experiments which I tried in the laboratory of the Royal Dublin Society in the year 1837, having had, through the kindness of Professor Davy, a galvanic battery of sixty pair of plates, five inches square, put at my disposal.

When fused phosphorus, sulphur, selenium and iodine, were submitted separately to the action of this battery charged with a strong acid solution, they conveyed the electrical current freely during the whole time, giving a spark whenever contact was broken; yet at the end of two hours they showed not the slightest trace of decomposition, no gas being evolved at either pole, which would have been the case had there been any water present.

Having by these experiments shown the direction of propagation of the electric force, I will now consider the source from which it originates in the voltaic pile, the mode of its transfer, and its sustaining principle.

Sir H. Davy's $\dagger$ opinion that the contact of the metals was the primum mobile of voltaic excitement, having been proved by Dr. Faraday $\ddagger$ to be erroneous, chemists are now pretty generally agreed that the electrical force developed in the voltaic pile is due altogether to chemical action, concerning which there are different opinions; of these, I will mention two, which are the most applicable to the present argument-Dr. Faraday'sई and Mr. Becquerel's $\|$. The former supposes that the development of electricity is due to decomposition alone, and in no case to the chemical union of bodies; while the latter contends that it is due to both, and in proof of his opinion shows that when an alkali unites with an acid, with a neutral salt, and in fact with any solution whose natural state is with regard to it electrically negative, a current of electricity will flow from the alkali to that solution. Sir H. Davy a different view of these experiments from Mr. Becquerel, supposing that the electric current is produced by the action of the acid or alkali upon the platinum plates; but the latter has shown that the electrical current is produced equally when

* Bakerian Lecture, 1809.

‡ Eighth Series, (880).

II Tom. ii. from page 77 to 81 .
+ Phil. Trans., Bakerian Lecture, 1826.

$\S$ Eighth Series, (927) (928).

if Phil. Trans., Bakerian Lecture, 1826. 
of the Electric Force traversing Interposed Media. 189

no such action could take place, the platinum poles being placed in separate cups filled with water*.

The accuracy then of Mr. Becquerel's experiments having been fully established, the question arises, how are we to reconcile them with other well-known contradictory facts? such as for instance those of $\mathrm{Sir} \mathrm{H}$. Davyt,-solid potash and sulphuric acid combining in an isolated platinum crucible, and causing no electrical development. Again, a plate of copper and of sulphur, when heated, have their electrical states increased until chemical action begins, when they cease.

The simplest and clearest course, and that most reconcileable with the laws of statical electricity, seems to me to be:to consider that no electrical development is caused by the union of an alkali with an acid, (the electricity being thereby disguised,) but that, at the instant before the union takes place, the particles of the alkali and of the acid, being in opposite electrical states, affect their surrounding particles by induction, causing thereby a feeble current of electricity to circulate from the acid through the galvanometer to the alkali, which supposition is borne out by the fact, that a dry acid and alkali, when in contact, show opposite electrical states.

The same arguments apply equally well with regard to thermo-electricity. The contact of two metals produces in them opposite electrical states. Their chemical union in an isolated vessel gives no electrical development; thus a "solid amalgam of bismuth and lead become liquid when mixed together, without producing any electrical effect:." Again, "a thin plate of zinc placed upon a surface of mercury, and separated by an insulating body, is found to be positive, the mercury negative; but when kept together a sufficiently long time to amalgamate, the compound gives no signs of electricitył."

These experiments explain why the contact of the two extremities of metallic wires, constituting a closed circuit, should, as the potash and nitric acid just mentioned, produce an induced electric current. That the electric states of different metals in contact, when excited by heat, do not follow the law of their natural electrical states, and change on increase of temperature, is no argument against the explanation $I$ have given, for upon what this change in the electrical excitation

* He might have added another experiment, free from all objectionsnamely, the increased intensity consequent upon an increased number of alternations of acid and alkali.

$\dagger$ Phil. Trans., Bakerian Lecture, 1807.

$\ddagger$ Ibid. 
produced by heat depends, whether upon a peculiar arrangement of the crystalline parts of the metal, or of their compound elementary particles, we are as yet perfectly ignorant.

That the same general law of the contact of metals and of fluids applies equally (although in an inferior degree, owing to their want of conducting power) to the contact of the gases, may be shown by the experiment of Dr. Faraday (Sixth Series) of the union of hydrogen and oxygen by a plate of platinum; the electrical force, which circulates by the interposed platinum plate, facilitating the union of the two gases*.

To return to the source of the voltaic force in the battery. Zinc, when placed in contact with a dry acid, has been found to become positively electrified. When the zinc plate has been immersed in the acid solution, being positive, it attracts oxygen, by union with which its electrical state is disguised, while the hydrogen, set free in a highly positive electrical state, reacts upon the oxide of zinc, rendering it negative by induction. The platinum wire connecting the positive solution with the negative zinc plate, reduces all for the moment to a state of equilibrium, so that the electricity becomes disguised, not transferred bodily from the platinum to the zinc; which state of equilibrium is no sooner restored than it is destroyed, the zinc regaining its positive state, and the oxide being removed by the acid.

If we consider then what takes place, we shall perceive that the zinc plate undergoes alternate states of induction and equilibrium, as do likewise the particles of the solution between the zinc and platinum plates, and, in fine, the platinum plate itself, and that as the number of alternations of zinc and platinum increases, the electrical energy of the zinc plate increases, as does also the rapidity of its oxidation and deoxidation, and as a consequence the rapidity of change of induction and equilibrium upon which the intensity of the current depends.

The decomposition of the electrolyte may be considered to be the effect produced by two forces acting upon its particles; the attraction of the polest of the battery (whether they be

- Aqueous solutions of different gases, when brought into contact, have been found to produce electrical currents.

+ In place of poles, I should more properly have said electrodes, their bounding surfaces. It follows, as a consequence of the theory, that the particles of oxygen in contact with the electrodes should be attracted by, and set free from, those electrodes upon each alternation of the states of induction and equilibrium; and that, when the induced state has not sufficient energy to overcome the affinities already engaged, the current of electricity passes without producing electrolyzation. For a different explanation, vid. Dr. Faraday's Series of Researches, 493, 494, 495, 534, 535, $536,537,807$. 
metal, water, or air) originating, while the electrical states induced upon the particles give the direction to the electrolytic action.

From what has been said above, we may, I think, presume that an electric current originates in a natural electro-inductive power of bodies when brought into contact, and is continued by alternate states of induction and equilibrium, the rapidity of change of state constituting its intensity. And inasmuch as the accumulation of the electric æather on the surface of the particles by the inductive force, and its recession on each return to a state of equilibrium produces what may be called an oscillation in the æther, the theory may be otherwise stated thus:--the mass of oscillating æether which surrounds the particles constitutes the quantity, while the rapidity of the oscillations constitutes the intensity of an electric current.

The late experiments of Dr. Faraday upon induction (Eleventh Series) showing that an insulated body (the particles of bodies may be presumed to be such) cannot receive an absolute charge of electricity, but only an inductive charge, afford a strong argument in favour of my views.

The theory proposed in this paper, and deduced from the experiments of Sir H. Davy, given in his Bakerian Lectures, is an extension of the views therein developed, reconciles the contact with the chemical theory, and reduces to the laws of statical electricity all the phænomena of electricity in motion. I will now endeavour to show how the law of the definite nature of electro-chemical decomposition, so beautifully developed by Dr. Faraday, follows as a consequence from this theory. Were the particles of all bodies endued with the same quantity of electricity, and of the same density, it is evident from the laws of statical electricity, that no one body conld have an attraction or repulsion for another; consequently, it is an evident fact, that the quantity and density of the electric æther varies in different bodies; and as, from the theory above stated, electricity never leaves the particles, but merely (to use the words of statical electricity) accumulates upon the surface, and returns, it follows that the electrical states of the particles of bodies are constant and unalterable, and therefore it is obvious that the law discovered by Dr. Faraday follows as a consequence from this hypothesis, which is at once clear and simple, which includes all the phænomena, and is but a reference of the laws of statical electricity to the particles of bodies in place of their masses. 